\title{
How to Manage your Supply Network to get better results
}

\author{
R. Hieber, D. Brütsch, F. Frigo-Mosca \\ Institute of Industrial Engineering and Management \\ Swiss Federal Institute of Technology (ETH) Zurich \\ (Switzerland), Domain of Prof. Dr. Paul Schönsleben \\ Tel: ++41-1-632 05 26, Fax: ++41-1-632 1040 \\ hie@bwi.bepr.ethz.ch
}

\begin{abstract}
The paper aims at providing a network diagnosis tool for the management of supply chains which can be very successful in evaluating and optimizing the performance of supply networks. Therefore, first of all, this paper gives an indication how variances within a supply chain can create great disturbances in the material flow over the own interorganizational boundaries. Analyses have shown that the standard deviation of the material flow of representative A-products can raise manifold along the supply chain. By investigating this phenomenon, also called the bull-whip effect (Lee, 1997), schedule instability was discovered as the root cause. To manage networks and to control the material flow over various links of the supply chain, a methodology, called network diagnosis system, has now been developed. Experiences with the application of this methodology have shown that not only the variances can be diminished, but also the cooperation between the different organizations can be significantly improved. Moreover, first results have also revealed, that the awareness of the involved members about the subsequent consequences and effects of local single actions within the supply chain is one of the most critical factors for a successful network management.
\end{abstract}

\section{Keywords}

Logistics, Supply Chain Management, Perfomance Measurement, Network diagnosis 
Nowadays, most of the companies are confronted with a rapid and radical change of the market conditions. Some of the most important tendencies are:

- Increasing internationalization of economical relations.

- Shifting from a vendor- to a customer-oriented behavior of the markets.

- Increasing complexity of products and services.

Hence, the future competitiveness of companies depends very much on their ability to adapt rapidly to changing market conditions. In a first step, the companies have already started optimizing their own logistics processes, by implementing concepts like BPR, JIT or TQM to get faster and more flexible. But the potential of improvements is limited, because most of the companies cover only a small part of the whole value chain. As a consequence, a second step must be initiated to look at the whole value chain to achieve the overall optimization of the supply chain (Schönsleben, 1998). Therefore, the organization, design and control of the whole value chain are becoming more and more essential for the success of the companies on the global markets (Wildemann, 1996; Towill, 1997). Interorganizational concepts, like extended enterprise models (Frigo-Mosca, 1995), Enterprise Resource Planning (ERP-systems) and SCM are now getting the attention of the management and research and extent the focus towards ever larger systems.

\section{OBJECTIVES AND METHODOLOGY}

The goal of this study was to improve the information and material flow between the organizations within a supply network. As a consequence, the transparency of the shared information among the partners should be optimized and hence, the ability to react on changing demand conditions should be increased.

Based on a case study in the automotive industry, first, the current situation and the existing issues in a supply chain network have been investigated. The analyzed network consisted of an Original Equipment Manufacturer (OEM), a system provider and its main supplier whereby the selected product group and related components can be best described as a high volume production with almost no product variation. All companies are located in different countries in Europe which already demonstrates that the control of the supply chain is a concern of all participating units.

First results of the supply network analysis have revealed that the main issues were poor delivery performance of the suppliers and a lack of an efficient inventory and material flow control. Furthermore, no appropriate supply chain performance measurement for evaluating the network existed.

To cope with these issues, the following methodology was developed which should enable the management to control the flow of materials and information 
over the critical interfaces by increasing the transparency and to define strategies to optimize the whole supply chain.

\section{THE CONCEPT OF A NETWORK DIAGNOSIS SYSTEM}

In order to gain more transparency into the information and material flow over the own organizational boundaries, the network diagnosis system covers the whole value chain, that means, from the raw material to the end user product. The goal must be to provide all critical and essential information for all involved organizational units for a successful management of a supply chain.

However, experiences have shown, that this ambitious goal can only be reached by implementing such a system step by step. Hence, the diagnosis system takes as a basis an hierarchical structure, which allows to define and adapt each subsystem to the current needs and situations. Figure 1 shows the basic structure of such a system.

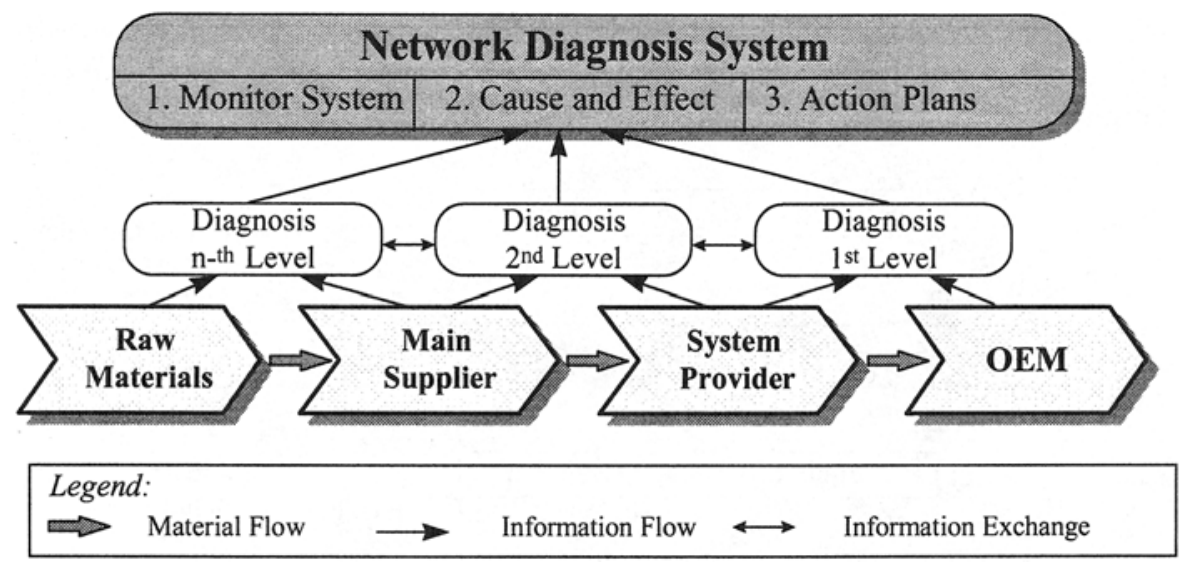

Figure 1 Structure of a Network Diagnosis System.

To reveal and communicate all essential information among the suppliers and customers, each subsystem consists of three elements:

- Monitoring system - data analysis

- Cause and effect relationship analysis

- Action plans

The purpose of these elements can be briefly described as follows: the monitoring system provides all critical performance measurements of all involved organizational units. By analyzing and monitoring graphically these data, charts and figures 
of the performance of the whole supply chain become visible. They build the framework for the following cause and effect relationships analysis among the supply chain group. Based on these results, weak spots and areas of improvement are identified, which, in a last step, can be mainly eliminated by focused action plans in co-operation with the external organizations.

Based on examples and experiences gained by the case study, all three elements of the diagnosis system will be described in detail. Thereby, the examples focus on one subsystem of the diagnosis system, which has already been implemented.

\subsection{Monitoring System}

By applying the diagnosis methodology, the first step is to collect, analyze and especially show graphically expressive data about the network performance. The most significant performance measure for analyzing networks is simply the material flow percentage of representative A-products. Therefore, bill of materials and operation plans have to be taken into account for following and especially calculating the number of outgoing and incoming components and products over the interorganizational boundaries. In this case, A-components (textiles), A-parts (cabin panels) and final products (cabin interiors) were analyzed in relation to their average usage.

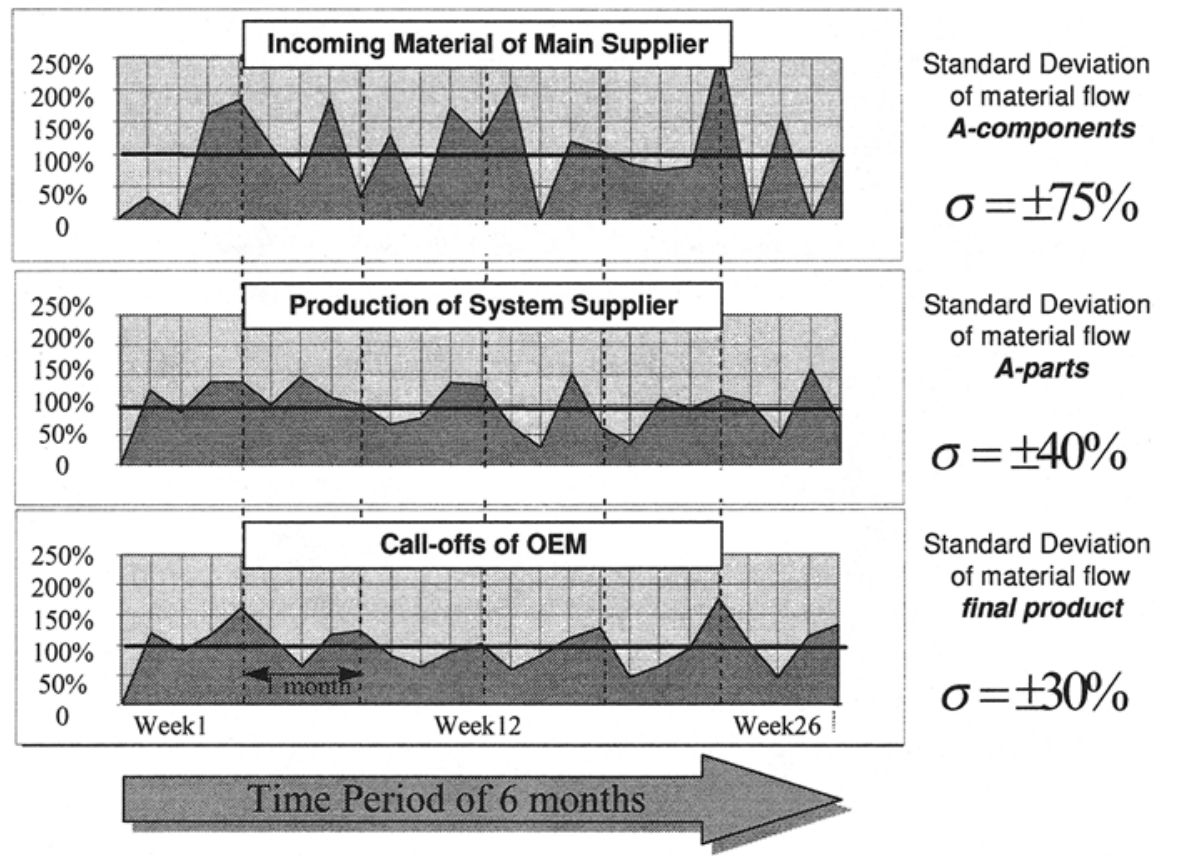

Figure 2 Variation up-stream the supply chain. 
Data was collected over a period of six months of all three involved units. Even in this short period of time, amazing results about system behavior have been discovered. The variation of call-offs, production and incoming material is growing constantly up-stream (see figure 2).

Even if the number of call-offs is nearly stable and the production mostly follows these order patterns, the standard deviation of the flow of incoming material is twice as high as the actual number of call-offs.

These very straightforward analysis with the resulted charts were then the basis for the first supply chain management group discussion.

\subsection{Cause and Effect Relationship Discussion}

By just monitoring and presenting these charts to the management of the involved units within the supply chain, a very successful "cause and effect explanation" analysis and discussion was started. Every involved unit, such as sales persons, production planners and purchasing personnel of the companies, got their homework to do. Additional information was collected and then again discussed within the supply chain group.

As main causes were then commonly identified:

- customer unpredictability;

- supplier unreliability;

- MRP-System.

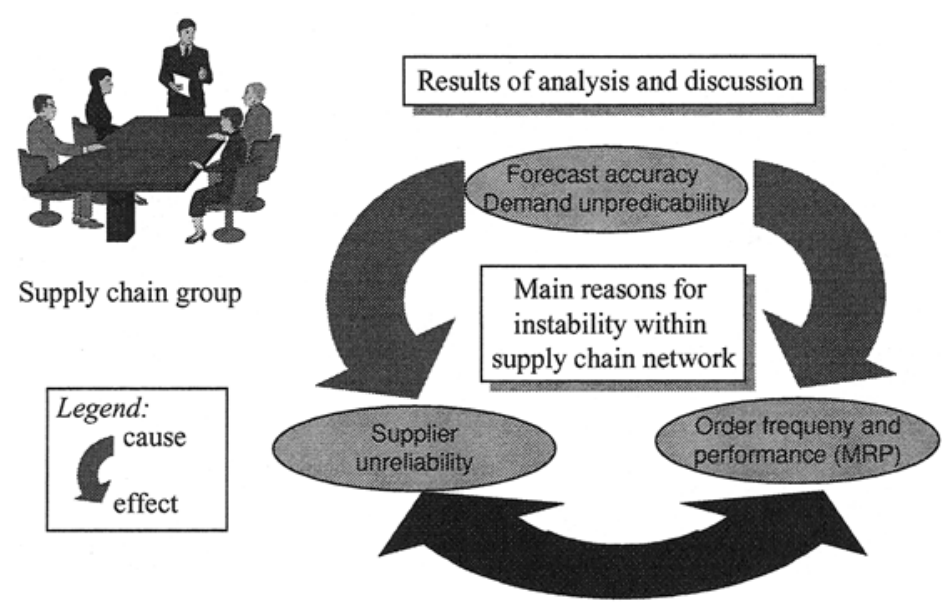

Figure 3 Reasons for Instability within supply chain networks.

First of all, the three major areas cannot be seen as totally uncorrelated objects. All of them are related to each other (see figure 3). Based on unexpected and 
frequent changes in the forecast of predicted call-offs, the MRP-system either generates or cancels orders, which effect tremendously the next member of the supply chain. In our case, this "system nervousness" was especially increased by the EDI-link between the MRP-system of the companies. As a result, the supplier performance is getting worse and just through excess inventory and safety buffers, the delivery schedule can be achieved.

\subsection{Action Plans based on first diagnosis results}

The first recommendation in this case study was to get control over the variations in the material flow within the supply chain. By just starting the discussion among the management of the supply chain group and presenting their frequent changing forecast schedules, they realized the need for modifying the system. Special variation limits within the planning horizon of the forecast can turn the supply chain into a more stabile system. Advantages are on both sides; on the one side, suppliers can reduce their safety buffers and improve their delivery performance, on the other side, initiated by the gradual change in the company culture, the OEM realizes that a cost reduction within the supply chain will also effect the costs and the price of its own final product. Figure 4 shows an example of a possible variation funnel for a demand schedule.

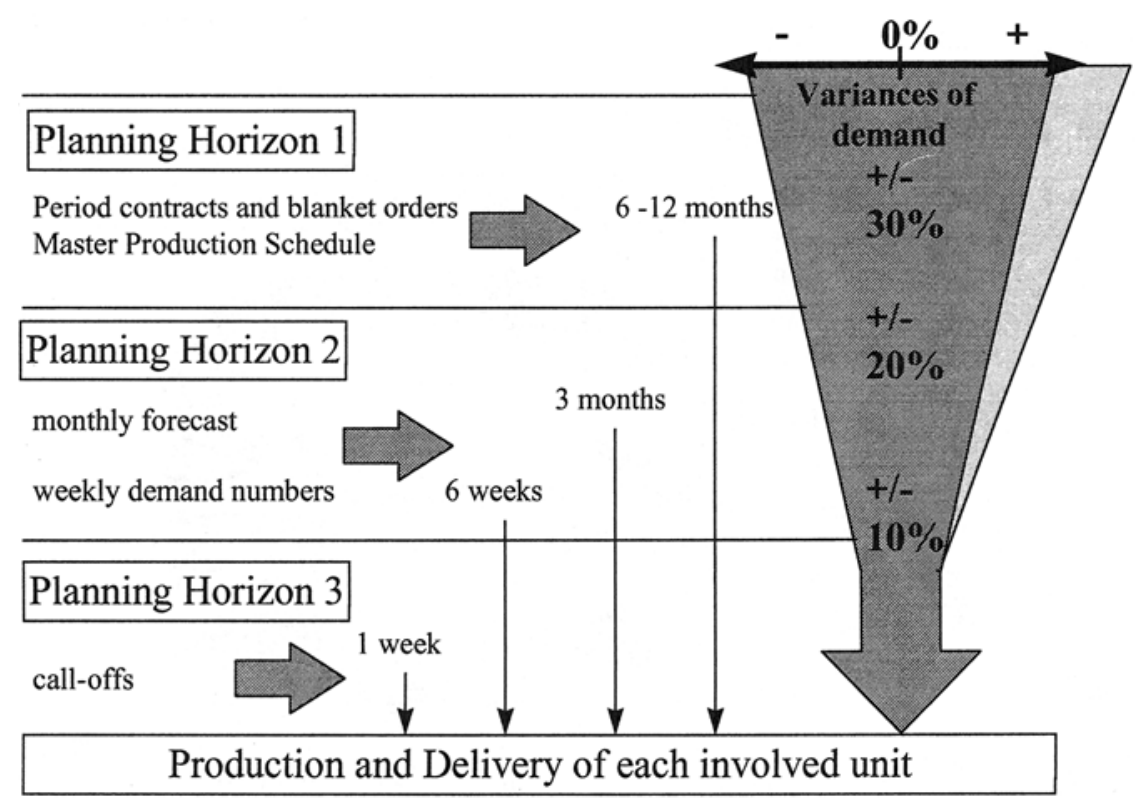

Figure 4: Variation funnel for demand schedule 
Further recommendations which resulted from the cause and effect analysis, sorted by the time horizon of realization:

- Improved and modified Supplier Evaluation System.

- Kanban Planning instead of MRP-System.

- Improved forecast methodology.

- Establishing Network Diagnosis System along the whole supply chain.

One of the most significant advantages of this methodology is, that by just sharing and discussing the collected data and charts among the management of the supply chain group, the understanding and perception for the issues of the other members of the chain can raise manifold. As a consequence, the management within the supply chain can learn that isolated actions, e.g. changing the demand schedule by the production planner of the OEM, can have tremendous impact on other areas upstream. Hence, it is very important to enlarge the diagnosis to a larger system to provide all chain members with the critical information, which they need in order to reach the goal - to get better results.

Frigo-Mosca, F. (1995) Advanced Logistic Partnership. Proceedings of the 1995 World Symposium of Integrated Manufacturing. APICS, Auckland, NZ, pp.31-35.

Lee, Hau, Padmanabhan, V. and Whang, Seungjin (1997) The Bullwhip Effect in Supply Chains, in Sloan Management Review, pp.93-102, Stanford.

Schönsleben, P. (1998) Integrales Logistikmanagement, Kapitel 2, Springer Verlag, Berlin.

Towill, D. (1996) The seamless supply chain - the predator's strategic advantage, in Int. J. Technology Management, special Issue on Strategic Cost Management, Vol.13, No.1, pp.37-56.

Wildemann, H. (1996) Netzwerkstrukturen als neue Form der Unternehmensorganisation, in: $Z W F$ 91, 1-2, S. 12-16. 


\section{Ralf Hieber:}

Born in 1971, Studies in Mechanical Engineering with the major in Manufacturing Systems at the University of Stuttgart (GER), Graduation (Dipl.-Ing.) in 1997, complementary studies of Industrial Engineering as a scholar at the University of Wisconsin-Madison (USA), 1996 graduation (M.Sc.) in Manufacturing Systems Engineering. Since 1997, scientific assistant of Prof. Dr. P. Schönsleben at the BWI of ETH Zurich with the research areas: Organization and Management of Production Network Structures and Virtual Enterprise.

David Brütsch:

Born in 1969. Studies at the ETH Zurich and graduated in Management and Production Engineering (dipl. Ing. ETH) in 1995. Since spring 1995, he works as a scientific assistant at the BWI of ETH Zurich with following research topics: Supply Chain Management, Business Process Reengineering, Network Logistics and Virtual Organizations.

Fabio Frigo-Mosca:

Fabio Frigo-Mosca, Dr. Sc. Techn. ETH, was born 1966 in Locarno (Ticino), Switzerland. From 1992 to 1996, he worked as Research Assistant at the Institute for Industrial Engineering and Management (BWI) of the Swiss Federal Institute of Technology Zurich. Since 1997, he is Senior Assistant and Head of the research team "Supply Chain Management and Virtual Organization" in the group of Prof. Dr. Paul Schönsleben at the BWI. Since Spring 1996, he works also as Senior Consultant for AFG \& Partner, a Swiss consulting company, in the areas of Total Quality Management and Supply Chain Management. 